\title{
A behavior systems view of responding to probe stimuli during an interfood clock
}

\author{
KATHLEEN M. SILVA and WILLIAM TIMBERLAKE \\ Indiana University, Bloomington, Indiana
}

\begin{abstract}
Two experiments used a behavior systems approach to relate the form of responses during an interfood clock to the temporal distance of the individual clock stimuli to food. Stimuli proximate to food should better control a focal search mode and related responses, whereas stimuli temporally distant from food should better control a general search mode and related responses. Experiment 1 conditioned two groups of rats with a sequence of four equal-length 12-sec clock stimuli that terminated with food and then tested for the conditioning of a general search mode by presenting an unconditioned moving probe stimulus (either a rolling ball bearing or a rotating mechanical door) during each of the clock stimuli. Consistent with a behavior systems view, contact with the ball bearing was markedly greater during a clock stimulus distant from food. The absence of similar differential contact of the door across the clock stimuli showed that the effect was specific to the ball bearing rather than a general response to stimulus dimensions of movement and sound. Experiment 2 showed that the general search mode was controlled by the clock stimulus rather than the passage of time.
\end{abstract}

Many studies have shown that animals display a sequence of behavior across intervals between regular presentations of reward. For example, when exposed to periodic presentations of food, pigeons systematically distribute their behavior across the interfood interval (IFI): Movement away from the feeder occurs after food, followed by a gradual increase in behavior directed at the feeder wall as the time of the next reward approaches (Staddon \& Simmelhag, 1971; Timberlake \& Lucas, 1985). More recently, researchers have attempted to gain precise temporal control of the distribution of responding during an IFI by using an interfood clock, a sequence of distinguishable external stimuli that fill the IFI. For example, Palya (1985) exposed pigeons to an interfood clock during a fixed-time (FT) 60 -sec schedule. The clock partitioned the IFI into ten 6-sec periods, and each period was correlated with a distinctive color on the response key. With this procedure, accelerated keypecking began about midway through the IFI and increased during stimuli successively closer to food (see also Dinsmoor, 1994; Dinsmoor, Dougan, Pfister, \& Thiels, 1992; Palya \& Bevins, 1990).

Interfood clocks have also been used to examine the control of multiple responses across an IFI (Matthews, Bordi, \& Depollo, 1990; Matthews \& Lerer, 1987). For example, Matthews and Lerer showed that pigeons exposed to a keylight that became increasingly brighter across the IFI developed a consistent distribution of responses be-

This study was facilitated by Grants NSF IBN 9408366 and IBN 9121647. Address correspondence to K. Silva, who is now at the Department of Psychology, University of Redlands, P.O. Box 3080, 1200 E. Colton Ave., Redlands, CA 92373-0999 (e-mail: ksilva@uor.edu), or W. Timberlake, Department of Psychology, Indiana University, Bloomington, IN 47405 (e-mail: timberla@indiana.edu). tween food presentations. Circling away from the feeder area (i.e., "retreat") predominated after food presentations when the keylight was dimmest. Pacing along the chamber walls predominated in the middle of the IFI, when the keylight was of a medium intensity. Pecking the key predominated just before food presentations, when the keylight was brightest. Subsequent tests that changed the order of the keylight intensities confirmed that keypecking and retreat were controlled by the keylight intensity rather than by the temporal interval following or preceding reward (see also Matthews et al., 1990).

An important question raised by this research concerns the origin of the responses occurring during the interfood clock. Matthews and Lerer (1987) interpreted interfood behavior as a set of Pavlovian conditioned responses (CRs). Keypecking during the keylight intensity just before food presumably developed through stimulus substitution. Retreat and pacing, through repeated pairings, became conditioned to the clock stimuli during which they initially occurred. Palya (1993; see also Palya \& Bevins, 1990) suggested that the placement of food in time establishes a gradient from the point of maximum imminence immediately preceding food to the point most removed from subsequent food presentations. This gradient is presumed to control behavior appropriate to the relative proximity to food. The beginning of the interval controls avoidance and low rates of behavior typical of that time period; the end of the interval controls high levels of approach behavior. Consistent with this view, stimuli correlated with later portions of the second half of the IFI support successively higher rates of keypecking that result in the production of the stimulus, whereas stimuli in successively earlier portions of the first half of the IFI support higher rates of keypecking that result in removal of the stimulus (Dinsmoor, Lee, \& Brown, 1986; Palya, 1993). 
Both accounts posit that each clock stimulus controls responses that are present during their portion of the IFI. However, these accounts do not predict the form of the responses that should be present initially, nor are they clear about why only some of the responses present during a clock stimulus are conditioned. The behavior systems framework (Timberlake, 1994; Timberlake \& Lucas, 1989; Timberlake \& K. M. Silva, 1995) attempts to predict the form of responses that emerge during an IFI by using an ethological approach relating the form of the CR to a sequence of appetitive search modes related to the temporal distance between the conditioned stimulus (CS) and the unconditioned stimulus (US). In the case of feeding, the behavior systems view postulates that a sequence of overlapping search modes (general search, focal search, handling/consuming, and postfood search) is organized by the predictable presentation of a US. Each mode is characterized by a repertoire of stimulus sensitivities and response components. The portion of the rat's feeding system described below represents a set of hypotheses and assumptions based on naturalistic observations and experiments of many researchers (Barnett, 1975; Ewer, 1971; von Steininger, 1950; Telle, 1966; Timberlake \& Washburne, 1989).

Near the beginning of a predatory sequence, a rat is assumed to be in a general search mode, characterized by attention to, chasing of, and contact with novel and moving stimuli that potentially signal prey. When predictors of imminent food are encountered, the animal enters a focal search mode in which responses are related to the immediate procurement of prey and involve interaction with a particular prey-associated location. Once the prey has been subdued, the animal enters a handling/consuming mode in which responses are related to immediate ingestion of the prey. Following ingestion, the hungry animal enters a postfood search mode related to localized search around the food site for further food items, and, in the absence of further food, the animal returns to a general search mode.

In the laboratory, the strength of different search modes and their accompanying responses have been evoked by using a rolling ball bearing, which mimics some characteristics of prey (e.g., size and motion), as part of or following conditioning with food (K. M. Silva \& Timberlake, 1997; Timberlake, Wahl, \& King, 1982; Timberlake \& Washburne, 1989). Because attention, approach, and initial contact occur before nosing in the feeder, we used these responses as assays of general and focal search, respectively. Handling/consuming responses occur once the food arrives.

According to the behavior systems view, the extent to which a well-trained CS controls a particular mode should relate to its temporal separation from the US. Temporally distant CSs are more likely to evoke a general search mode and related behavior, whereas temporally proximate CSs are more likely to evoke a focal search mode and related behavior. Matthews and Lerer's (1987) study is consistent with the behavior systems view: Retreat and pacing away from the food site after food can be viewed as general search activity related to searching for food, whereas keypecking late in the IFI is a more focal search response related to the imminent procurement of food (Timberlake \& K. M. Silva, 1995; see also Papadouka \& Matthews, 1995).

Recently, K. M. Silva and Timberlake (1998) examined the effect of a periodic interfood clock on multiple responses in rats. During an interfood clock composed of a sequence of four 12-sec lights predicting food (S1-S2S3-S4-food), rats engaged in a sequence of responses consistent with the behavior systems view: Activity near the feeder (paw grooming and rearing), a measure of postfood focal search, peaked during Sl after food presentations. General search behavior, in the form of locomotion away from the food site, peaked during S2. Focal search behavior, in the form of nosing in the feeder, peaked during S4. This sequence of responses occurred during IFIs ranging from 12 to $192 \mathrm{sec}$. Furthermore, the control of the behaviors during the second and fourth clock stimuli was apparently scalar. That is, locomotion away from the food site peaked (i.e., reached a maximum level of responding) during S2, and nosing in the feeder peaked during S4 during all IFI lengths. Conversely, the responses (rearing and paw grooming) occurring during the first clock stimulus were time bound, peaking at the same absolute time since food was presented regardless of IFI length. Thus, it appears that for some responses, a temporal rescaling of responding occurs over a wide range of interval lengths. It has also been shown that appetitive sequences observed during relatively short intervals between food presentations resemble that observed in more naturalistic settings (see Timberlake \& K. M. Silva, 1995; Timberlake \& Washburne, 1989).

The purpose of the present experiments was to further explore the contribution of search modes to responses that occur during an interfood clock by testing the ability of clock stimuli to facilitate approach to and contact with the unconditioned probe stimulus of a rolling ball bearing. That a clock stimulus presented temporally distant from food should condition a general search mode and evoke attention to, approach to, and contact with an unconditioned ball bearing, whereas a clock stimulus presented temporally close to food should condition a focal search mode and feeder-related response, is consistent with a number of studies. For example, Timberlake et al. (1982) showed that the general search responses of approach to and initial contact with a predictive ball bearing CS occurred only with relatively long CS-US intervals. On the other hand, at shorter intervals, the rats increased nosepoking in the feeder in the presence of the ball bearing. More recently, using contact with an unconditioned rolling ball bearing to assess the strength of a general search mode, K. M. Silva and Timberlake (1997) showed that a long-duration tone-CS increased contact with a rolling ball bearing above a baseline in which the ball bearing was presented alone. Conversely, a short-duration CS facilitated nosing in the food tray rather than ball-bearing contact. On these grounds, we expected an unconditioned 
ball-bearing probe presented during individual clock stimuli to evoke attention, approach, and initial contact primarily during the middle clock stimuli. On the basis of differential facilitation of locomotor behavior during the S2 clock stimulus in K. M. Silva and Timberlake (1998), which we interpreted as based on a general search mode, we expected maximum response to the ball bearing during the same clock stimulus.

In Experiment 1, two groups of rats were exposed to an interfood clock in which a sequence of four 12-sec stimuli was followed by food (S1-S2-S3-S4-food). Following training, an unconditioned probe stimulus was presented (on separate trials) during each clock stimulus. For one group, the probe stimulus was a rolling ball bearing. For the other group, the probe stimulus was the opening of a rat-sized mechanical door. On the basis of the behavior systems framework (Timberlake \& Lucas, 1989; Timberlake \& K. M. Silva, 1995), we hypothesized that a temporally distant clock stimulus should better condition a general search mode and that it would be displayed most clearly by increased contact with the ball bearing rather than the moving stimulus door. In Experiment 2, we attempted to clarify further the control of the elevated levels of ball-bearing contact obtained in Experiment 1 by training rats on an FT schedule of reinforcement without an interfood clock.

\section{EXPERIMENT 1 Responses to an Unconditioned Moving Probe Stimulus}

In this experiment, rats were first trained with an interfood clock in which a sequence of four 12-sec lights (S1, S2, S3, S4) flashing at different rates was followed by food. Following this initial training, either a moving ball bearing (Group BB) or a moving mechanical door (Group Door) was presented during the different clock stimuli. Findings from previous work (K. M. Silva \& Timberlake, 1997; Timberlake et al., 1982; Timberlake \& Washburne, 1989) suggested that the ball bearing is an unconditioned probe stimulus for a general search mode because it possesses dimensions of appropriate size and movement for eliciting prey-related responses in rats. We assumed that the amount of approach to and contact with a rolling ball bearing indicates the strength of the general search mode (Timberlake \& Washburne, 1989) and that rats should show markedly greater approach to and contact with the ball bearing when it is presented during S2 as opposed to the other clock stimuli. On the other hand, stimuli presented more temporally proximate to food are presumed to better condition a focal search mode, so rats should differentially nose in the feeder during those elements of the interfood clock just before food.

Group Door served to test the importance of the preyrelated qualities of the ball bearing in evoking responding. For Group Door, the unconditioned probe stimulus was a 9-cm-high $\times 14-\mathrm{cm}$-wide door located on the back wall approximately $26 \mathrm{~cm}$ from the feeder (approximately halfway between the place where the ball bearing emerged and the feeder). Preliminary work showed a level of unconditioned attention and approach to the moving door that was similar to levels shown to the moving ball bearing. If, as we hypothesize, a general search mode differentially facilitates contact with potential prey-related stimuli like the moving ball bearing, we would not expect a similar pattern of effects for the door. On the other hand, if a general search mode facilitates attention to any stimulus possessing dimensions of novelty, movement, and auditory stimulation, rats should approach and contact the door in the same pattern and amount as they contact the ball bearing. In either case, we would expect nosing in the feeder to be highest during S4 because of the conditioning of the strong focal search mode to that stimulus presented immediately before food.

An alternative set of predictions can be generated by assuming that no general search mode is conditioned to $\$ 2$. Instead, the eliciting qualities of the ball bearing and the moving door simply compete with the conditioned nosing in feeder response. On this view, responding to the ball bearing and door should be high during $\mathrm{S} 1, \mathrm{~S} 2$, and $\mathrm{S} 3$ because competition with nosing in the feeder is relatively low. Furthermore, previously measured baseline levels suggest that contact should reach a maximum of $25 \%$ to $30 \%$ of the trials (K. M. Silva \& Timberlake, 1997; Timberlake et al., 1982; preliminary data). This is the unconditioned level of approach and contact observed when the ball bearing and door are presented in the absence of food and other stimuli. Also, we would expect to find an inverse correlation between level of contact with the ball bearing or the door and the amount of nosing in the feeder.

\section{Method}

\section{Subjects}

Sixteen naive female Sprague-Dawley albino rats, approximately 90 days old at the start of the experiment, were maintained at $85 \%$ of their free-feeding weights throughout the experiment. Outside an experimental session, the rats were housed in individual home cages in a colony room regulated by a 12:12-h light:dark cycle. They were fed approximately $12 \mathrm{~g}$ of commercial rat chow in their home cages after each experimental session to maintain them at $85 \%$ of their free-feeding weight. Water was always available in their home cages. The 16 rats were divided into two groups (Group BB and Group Door) of 8 rats each. The groups were matched on weight.

\section{Apparatus}

A schematic of the apparatus is shown in Figure 1. Rats were tested in four identical chambers. Each chamber was a rectangular sheet-metal box $60 \times 30 \times 30 \mathrm{~cm}$ with a Plexiglas top and front. The food receptacle was $2.0 \times 5.0 \times 1.8 \mathrm{~cm}$ deep and located on a side wall $12 \mathrm{~cm}$ from the back wall of the chamber. Food reward was delivered by a Waltke feeder (Waltke Scientific Enterprises, Psychology Department, Indiana University), which delivered two 45-mg Noyes pellets into the feeder. An infrared beam projected across the entrance of the feeder, an $11.0 \times 10.0 \mathrm{~cm}$ platform was located on the floor in front of the feeder, and a $9.0 \times 14.0 \mathrm{~cm}$ platform was located on the floor along the back of the chamber. A retractable lever was located on the feeder wall $5 \mathrm{~cm}$ from the back of 


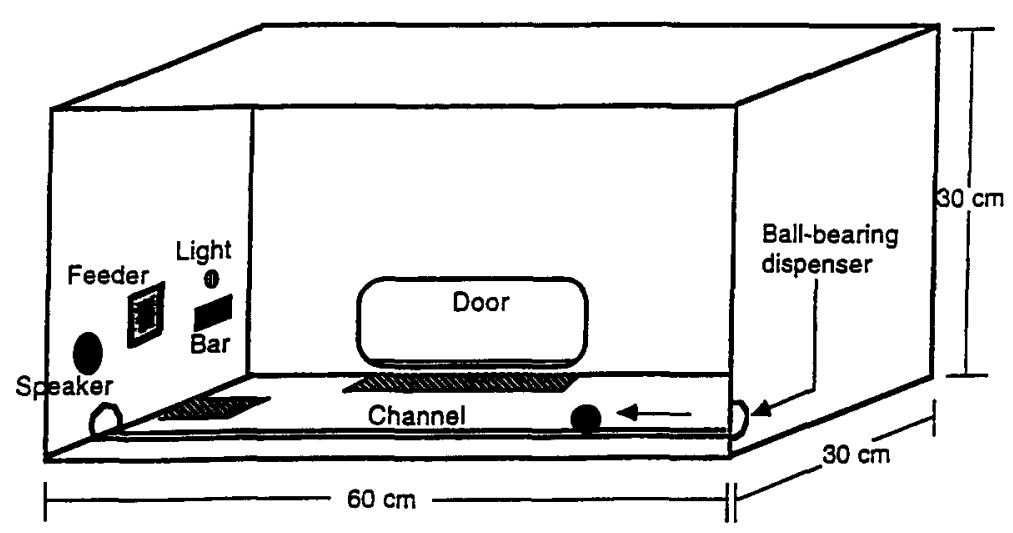

Figure 1. A schematic of the apparatus.

the chamber and $4 \mathrm{~cm}$ above the floor. The clock stimuli were presented on a green jewel light $2 \mathrm{~cm}$ above the lever.

Rolling ball bearings and the operation of a mechanical door served as probe stimuli in the test phase. A modified BRS feeder dispensed 1.6-cm ball bearings through a hole into a channel that ran the length of the apparatus into an exit hole located on the feeder wall. The channel and the floor of the apparatus were slanted approximately $6^{\circ}$ down from the entrance hole to the exit hole, and the floor was also slanted toward the channel so that a ball bearing dropped anywhere in the chamber reentered the channel and rolled out. The ball bearing rolled on two steel rods at the bottom of the channel. The channel and the entrance and exit holes were located $8.8 \mathrm{~cm}$ from the front wall. Once dispensed, a ball bearing took approximately $2 \mathrm{sec}$ to enter the chamber and $4 \mathrm{sec}$ to roll through it, if undisturbed.

The door, made of a rectangular piece of sheet metal $9.0 \times 14 \mathrm{~cm}$ and located on the center of the back wall $2 \mathrm{~cm}$ above the floor, protruded $2 \mathrm{~cm}$ into the chamber. When opened, the door rotated back behind the chamber wall to expose a compartment $9.0 \times 14 \times 5 \mathrm{~cm}$ deep attached to the chamber wall. The front of the compartment, which extended $2 \mathrm{~cm}$ into the chamber, had a wire grid cage on the front so that a rat could not climb in the compartment.

The experimental room also contained a video camera that monitored the experimental chambers. The video camera was connected to a Panasonic VCR that recorded experimental sessions and a Panasonic monitor for closed-circuit observation. Data logging, control equipment, and the VCR were located in an adjacent room. The chambers and the programming and recording equipment were located in separate rooms.

\section{General Experimental Procedures}

Experimental sessions were conducted at the same time daily, 6 days per week. There were 20 IFIs. Each session terminated immediately following the 20 th IFI. The experimental procedure consisted of four phases: chamber adaptation, feeder training, food delivery after fixed intervals of time, and a test phase.

Chamber adaptation. The rats were put in the chambers for 10 min on 2 successive days. No food or stimuli were presented. In their home cages, subjects were fed the same number of food pellets that they received during later phases.

Feeder training. Five pellets were placed on the platform in front of the feeder at the beginning of a session, and 20 pellets were placed in the feeder. A session terminated after the subjects had eaten all the pellets or after $30 \mathrm{~min}$ had elapsed, whichever occurred first. A rat was considered trained when it ate 20 food pellets within a 30 -min session for two consecutive days.
Interfood clock training. Both groups of rats were exposed to 16 sessions with an interfood clock consisting of a sequence of four 12-sec lights flashing at different rates. The first light (S1) did not flash; the second light (S2) flashed on:off at a rate of $1 \mathrm{sec}$; the third light (S3) flashed on:off at a rate of $0.5 \mathrm{sec}$; the fourth light (S4) flashed on:off at a rate of $0.1 \mathrm{sec}$. Two food pellets were presented immediately after the offset of the last stimulus (i.e., S1-S2-S3S4-food). To stimulate contact with the lever, it moved in and out of the chamber during stimulus changes. The present experiment did not counterbalance for the order of flashing rates. However, previous research has shown that the pattern of responses during the interfood clock is the same whether the clock stimuli flash progressively faster, progressively slower, or when the flashing rate is mixed (K. M. Silva, 1996).

Ball-bearing probe trials. After interfood clock training, Group BB rats received four successive test sessions in which eight probe trials (ball-bearing trials) were interspersed among regular trials of the basic interfood clock (basic trials). During probe trials, a ball bearing was presented during one of the four clock stimuli. A total of eight probe trials, two of each type (i.e., a ball bearing presented during $\mathrm{S} 1, \mathrm{~S} 2, \mathrm{~S} 3$, or $\mathrm{S} 4$ ), were presented during each test session. These trials ended in a $2-$ sec time-out during which the light from which the clock stimuli were presented was unlit, the lever was retracted, and no food was presented. Ball bearings were presented $4 \mathrm{sec}$ after the onset of a clock stimulus. Each session included 12 basic (i.e., reinforced) trials. The position of probe trials in a session was random; however, each session began with four basic trials, and at least one basic trial separated each probe trial. On the basis of previous data (see, e.g., K. M. Silva \& Timberlake, 1997), it was expected that differential ball-bearing contact might occur only on the first one or two test sessions. However, because the present design differed from those used previously, we conducted four test sessions in case the effect lasted longer than in previous studies. The present results showed that differential ball-bearing contact occurred only on the first test session. Thus, only data from this session are shown. The disappearance of differential ball-bearing contact after the first test session is likely due to probe trials being given during extinction (see also K. M. Silva \& Timberlake, 1997).

Door probe trials. After training, Group Door received test sessions in which eight probe trials (door trials) were interspersed among regular trials of the basic interfood clock trials used in training (basic trials). During probe trials, a door at the back of the chamber was opened during one of four clock stimuli. A total of eight probe trials, two of each type (i.e., door opened during S1, S2, S3, and S4), were presented during a test session. These trials ended in a 2 -sec time-out during which no food was presented. The door 
opened $4 \mathrm{sec}$ into each portion of the clock stimulus and closed $8 \mathrm{sec}$ later at the offset of the clock stimulus. Each session included 12 basic (i.e., reinforced) trials. The position of probe trials in a session was random; however, each session began with four basic trials, and at least one basic trial separated each probe trial.

Dependent measures. The duration of nosing in the feeder during each clock stimulus was measured for both groups. In addition, for Group BB, the percentage of trials with a ball-bearing contact (defined as touching the ball bearing with the nose, mouth, or paw within $4 \mathrm{sec}$ of the ball bearing entering the chamber) was scored from videotaped recording of the sessions. An interobserver agreement calculated on all trials with a ball bearing yielded a score of $91 \%$. For Group Door, the percentage of trials with a door approach and contact was scored from videotaped recording of the sessions. A contact was defined as touching the exposed grid cage with the nose, mouth, or paw within $4 \mathrm{sec}$ of the door opening. A rat was scored as approaching the cage when it walked toward the cage while moving at least three paws on the floor. An interobserver agreement calculated on all trials with a door operation yielded a score of $93 \%$.

We also wanted to obtain a measure of behavior directed toward the light. Since a lever was located below the light, we measured the duration of contacting the lever as an index of responding to the light. Previous research using a serial conditioning procedure showed that rats typically touch a lever located below a light CS when they approach the light (F. J. Silva, Timberlake, \& Koehler, 1996). The results of the present experiments showed that the rats rarely contacted the lever, and there were no significant differences across the interfood clock. Therefore, these data were not included in the results for any of the experiments.

\section{Results \\ Nosing in the Feeder Across the Interfood Clock: Training Versus Test}

To demonstrate that subjects behaved similarly during the interfood clock in training and test sessions, Figure 2 shows the duration per second spent nosing in the feeder during the interfood clock averaged over the last four training sessions compared with the first test session for Groups BB and Door. All statistical tests consisted of a repeated measures analysis of variance (ANOVA) that examined the influence of condition type (training vs. test) and clock stimulus (S1, S2, S3, and S4) on the levels of a particular dependent measure for each group. Statistically significant effects are reported at the $p<.05$ level.

Overall, both groups showed similar changes in nosing in the feeder during training and test: Nosing in the feeder increased during the last half of the IFI. A main effect of clock stimulus confirmed that subjects in both groups showed similar changes in responding across stimuli during training and test $[F \mathrm{~s}(3,21)>30.06]$. Scheffé tests conducted on the data from each group confirmed that during the training and test sessions, nosing increased from S2 to S3 and then further increased from S3 to S4. Also, there was more nosing during S4 than S1. For both groups, contacting the lever (data not shown) changed little across clock stimuli $[F \mathrm{~s}(3,21)<1.03, p>.10]$.

\section{Probe-Stimulus Contact}

The left graph of Figure 3 shows the percentage of trials in which Group BB rats contacted a ball bearing during each clock stimulus in the first test session. Subjects contacted the ball bearing more than twice as much dur-

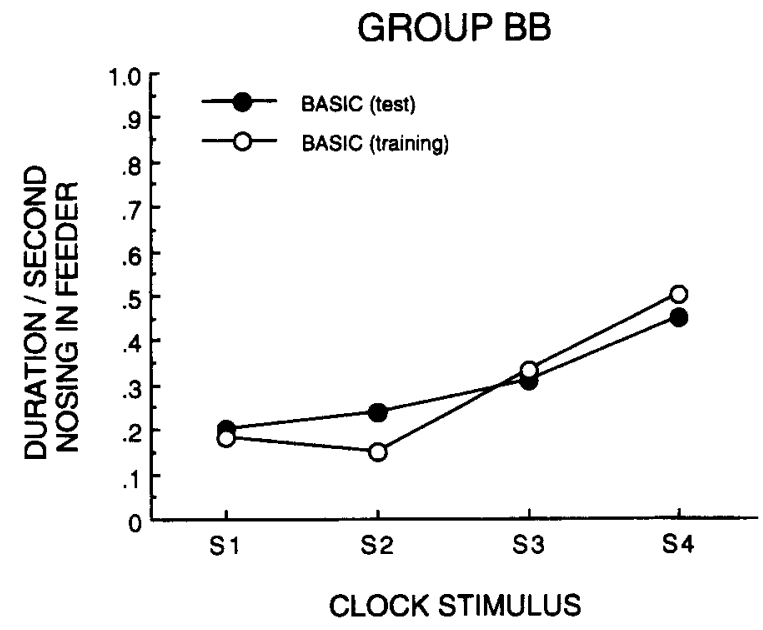

GROUP DOOR

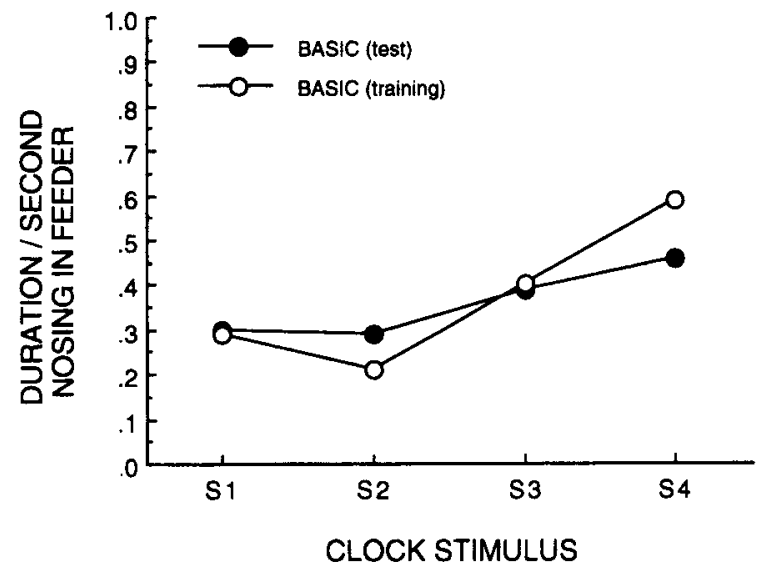

Figure 2. The mean duration per second spent nosing in the feeder averaged across the last 4 days of interfood clock training and during the basic trials of the first test session for Group BB and Group Door.

ing $\mathrm{S} 2$ as during the remaining clock stimuli (contact occurred on nearly $70 \%$ of trials during S2 as opposed to less than $30 \%$ during the other stimuli). This result was confirmed by a repeated measures ANOVA comparing ballbearing contact across clock stimuli $[F(3,21)=6.67]$ and a subsequent Scheffé test. During the remaining three test sessions (data not shown), the levels of ball-bearing contact were at baseline levels (near 30\%) during all clock stimuli.

The right side of Figure 3 shows the percentage of trials in which Group Door rats contacted (top graph) or approached (bottom graph) the cage during each clock stimulus in the first test session. Contacts did not differ during $\mathrm{S} 1, \mathrm{~S} 2$, $\mathrm{S} 3$, or $\mathrm{S} 4[F(3,21)<1]$. In the case of ballbearing contacts, rats nearly always contacted a ball bearing when they approached it. However, in the case of cage contacts, rats sometimes approached the cage but did not contact it. Thus, both approach and contact of the cage were measured. The results showed that neither approach nor contact of the door showed any significant differences 

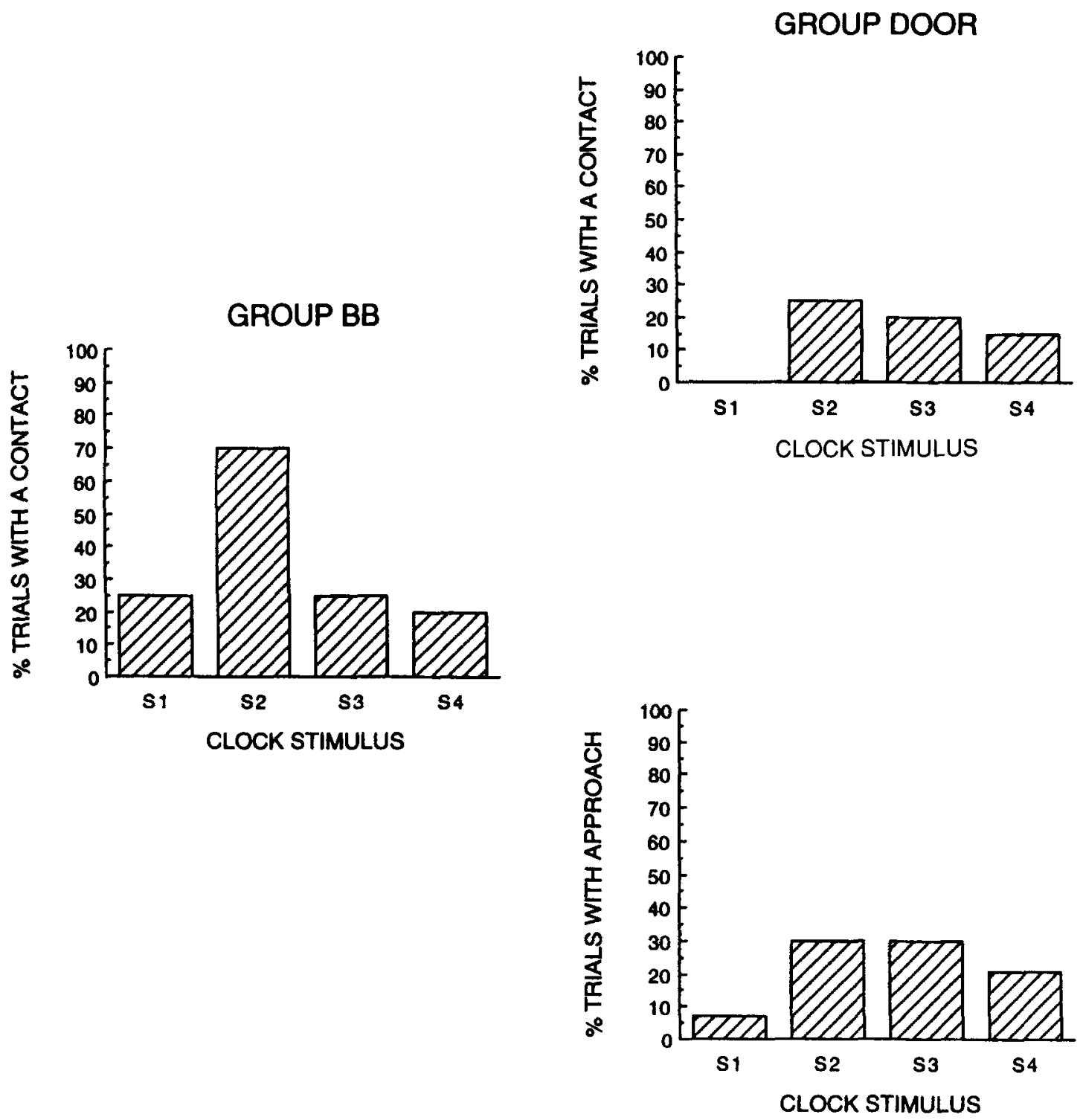

Figure 3. The percentage of trials with a ball-bearing contact (left graph) during each clock stimulus during test for Group BB and the percentage of trials with a contact (top-right graph) and an approach (bottom-right graph) of the cage during each clock stimulus during test for Group Door.

across clock stimuli $[F \mathbf{s}(3,21)<1]$. In addition, there was no differential approach or contact during the remaining test sessions (data not shown).

\section{Ball-Bearing Versus Basic Trials}

Figure 4 shows nosing in the feeder on the 1 st test day during the clock stimuli on which ball bearings were presented (ball-bearing trials) and compares it with nosing in the feeder from the same clock interval during trials on which no ball bearing was presented (basic trials). Because ball-bearing presentations generally decrease or do not affect other behavior, all statistical tests consisted of one-tailed correlated samples $t$ tests. For Group BB, the test compared responding during a ball-bearing trial with responding during the corresponding stimulus interval of a basic trial. Subjects spent less time nosing in the feeder during ball-bearing trials than basic trials for each clock stimulus $[t \mathrm{~s}(7)>2.35]$. Because Group BB's nosing in the feeder appeared more suppressed during $\mathrm{S} 2$ and $\mathrm{S} 3$ than during $\mathrm{S} 1$ and $\mathrm{S} 4$, suppression ratios were calculated for each clock stimulus. The ratios were obtained by dividing the duration of nosing in the feeder during portions of the clock stimulus in which the ball bearing was presented (i.e., the last $8 \mathrm{sec}$ of a clock stimulus on ball-bearing trials) by the sum of nosing during portions of the clock stimulus in which the ball bearing was presented and the same time period during basic test trials. The closer the ratio is to zero, the more responding 

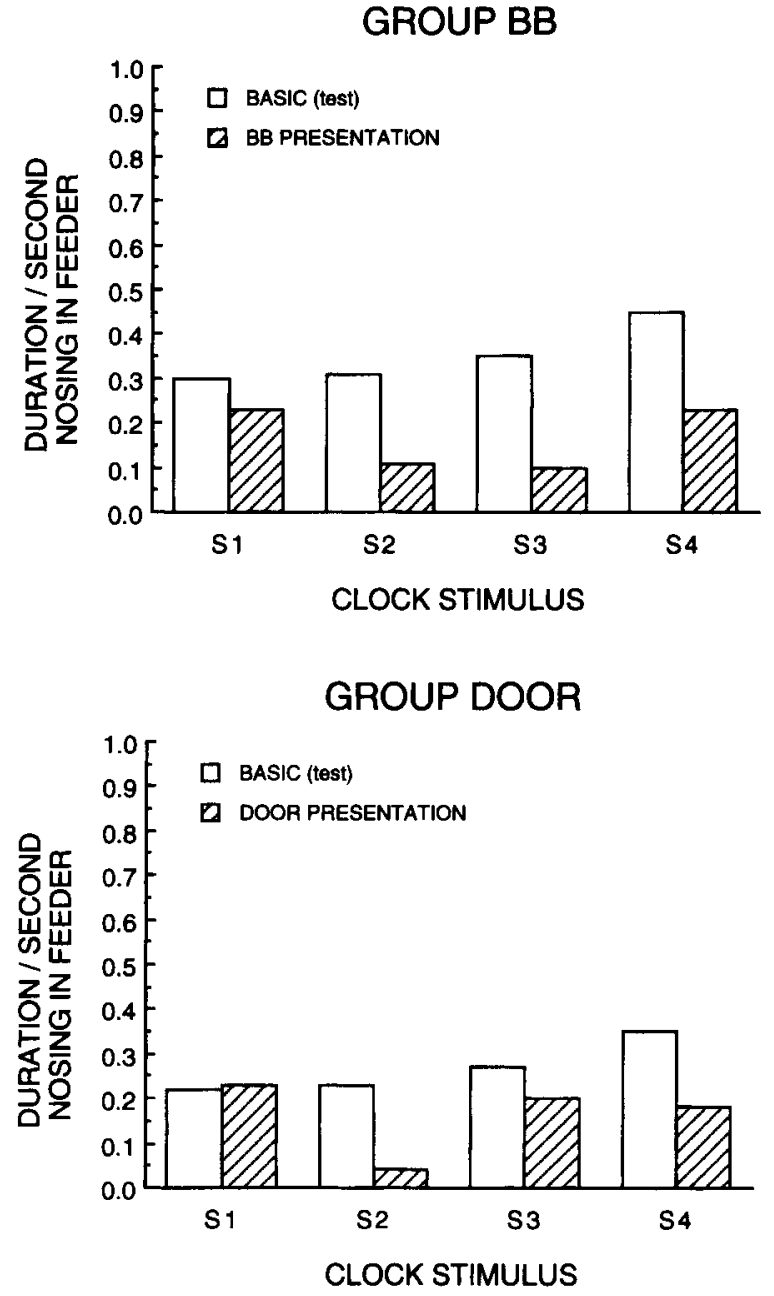

Figure 4. The mean duration per second spent nosing in the feeder from the portions of the clock stimuli in which ball bearings were presented (hatched bars) and from the same time period during basic trials (solid bars) of the test for Groups BB and Door. Because the ball bearings were presented $4 \mathrm{sec}$ after the onset of a clock stimulus for Group BB, and the door was opened $4 \mathrm{sec}$ after the onset of a clock stimulus for Group Door, the data shown were taken from the last 8 sec of each clock stimulus.

was suppressed. The suppression ratios for $\mathrm{S} 1, \mathrm{~S} 2, \mathrm{~S} 3$, and S4 were $.28, .18, .16$, and .25 , respectively. However, the differences among the ratios were not statistically significant $[F(3,21)<1]$.

For Group Door, the $t$ test compared responding during door-probe trials with the corresponding clock interval of a basic trial. Subjects spent less time nosing in the feeder when the door operated during S2 and S4 than during the $\mathrm{S} 2$ and $\mathrm{S} 4$ portions of basic trials $[t \mathrm{~s}(7)>2.77]$. The suppression ratios for Segments 2 and 4 were . 19 and .28, respectively, and the differences were not statistically significant $[t(7)<1]$.

\section{Discussion}

In sum, the results showed that ball bearings presented during S2 evoked considerably more contact than those presented during the other clock stimuli. In contrast, the moving door evoked the same (baseline) amount of approach and contact during each of the clock stimuli. These results are consistent with a behavior systems view that stimuli temporally far from food should come to control a general search mode that facilitates responses that are part of the rat's repertoire of general search behavior, such as approach and contact of a moving stimulus potentially resembling prey. The absence of facilitation of approach and contact of the moving door indicates that novelty, movement, and sound are not general dimensions of stimulation that facilitate a response. Instead, the response may be part of the animal's preorganized repertoire. In the case of the ball bearings, characteristics such as size and motion appear to be important. In other cases, other stimulus characteristics may be important (see Timberlake \& Grant, 1977, for results with social stimuli).

It might seem tempting to argue that the difference in responding to the ball bearing and the moving door was a matter of salience. However, preliminary work revealed similar levels of baseline contact of the ball bearing and the moving door, suggesting that their general salience was similar. An argument for a specialized salience is indistinguishable from the behavior systems view.

The present results also provided information about the extent to which nosing in the feeder and ball-bearing contact compete for expression. Recall that during the interfood clock, animals showed increased nosing in the feeder across the last half of the IFI (see also Lucas, Timberlake, \& Gawley, 1988; Matthews \& Lerer, 1987; Palya, 1985 ). Ball bearings equally disrupted nosing in the feeder during all clock stimuli, even though they evoked a substantial amount of contact during $\mathbf{S} 2$ only. If the rats simply contacted the ball bearing during S2 because of less competition from nosing in the feeder, there should have been an inverse relation between ball-bearing contact and nosing in the feeder, a result that did not occur. In addition, if levels of ball-bearing contact during $S 2$ were due to the diminution of response competition and not the conditioning of the clock stimulus, we should have observed contact with the ball bearing during $\mathbf{S} 2$ on no more than $30 \%$ of the $S 2$ test trials, the baseline level of contact in the present apparatus. However, contact occurred on nearly $70 \%$ of the $\mathrm{S} 2$ test trials, whereas contact occurred near baseline levels (30\%) during the other clock stimuli. Finally, if competition for expression was the primary determinant of $\mathrm{S} 2$ responding in Group $\mathrm{BB}$, a similar pattern of responding should have occurred in Group Door, an effect that did not occur.

\section{EXPERIMENT 2 Responding to Ball Bearings Without an Interfood Clock}

Although the results of Experiment 1 are consistent with the view that a general search mode is conditioned by presentation of food and that a rolling ball bearing can serve as an unconditioned probe for this mode, it did not decisively demonstrate that the clock stimulus, $\mathrm{S} 2$, controlled 
the general search mode. It might be argued that a general search mode was conditioned to other stimuli in the situation, such as the implicit temporal cues confounded with the clock stimuli and/or the presentation and withdrawal of the lever at the transition between each element of the interfood clock (see Palya \& Chu, 1996; Pavlov, 1927). In support of the importance of the passage of time as a conditioned cue, previous research has suggested that general search-related responses may be less sensitive to external cues than are focal search responses such as nosing in the feeder (Domjan, 1994; Matthews et al., 1990).

Experiment 2 trained two groups of rats on an FT 48$\mathrm{sec}$ schedule of reinforcement without an interfood clock. To minimize external cues predicting food, in one group (Group FT-Time), the lever came out at the beginning of the session and remained out for the entire session. In a second group (Group FT-Lever), the lever moved in and out of the chamber wall every $12 \mathrm{sec}$ (as in Experiment 1). The flashing lights that served as the clock stimuli in Experiment 1 were never presented. Following training, a rolling ball-bearing probe was presented during each quarter of the IFI, corresponding to the timing of the different clock stimuli in Experiment 1. If counting lever presentations is the critical predictive cue conditioning a general search mode, the results of Group FT-Lever should resemble those of Experiment 1 by showing much higher ball-bearing contact during the second quarter of the IFI, but group FT-Time should show little or no differential contact across the IFI. If implicit temporal cues are the important factors, both groups should contact more ball bearings during the second quarter of the IFI than in the other quarters. In contrast, if the clock stimuli in Experiment 1 are important, both groups should show little or no difference in responding to the ball bearing during the different quarters of the IFI.

\section{Method}

\section{Subjects}

Sixteen naive female Sprague-Dawley albino rats, approximately 90 days old, served as subjects. The 16 rats were divided into two groups (Group FT-Time and Group FT-Lever) of 8 rats each, and the groups were matched on weight.

\section{Apparatus}

The same apparatus as in the previous experiment was used.

\section{Procedure}

The same general experimental procedures, chamber adaptation, and feeder training as in the previous experiment were used. Following feeder training, the rats were first trained on an FT 48-sec schedule of reinforcement for 16 sessions. For Group FT-Time, the lever extended into the chamber at the beginning of the session and remained there until the end of the session. The lever was presented in this manner to minimize any cues that might predict the delivery of food. For Group FT-Lever, the lever moved in and out of the chamber wall at the beginning of each quarter of the IFI. After the fourth segment, the lever was retracted, and two food pellets were presented. After training, both groups received four test sessions in which eight probe trials (ball-bearing trials) were interspersed among regular trials of the basic FT trial. During probe trials, a ball bearing was presented during one of four segments of the IFI that corresponded to the same four segments occupied by the clock stimuli in Experiment 1. There were no clock stimuli used during training or test phases. A total of eight probe trials, two of each type (i.e., a ball bearing presented during Segment 1,2,3, or 4), were presented during the test session. These trials ended in a 2 -sec timeout during which no food was presented. Ball bearings were presented $4 \mathrm{sec}$ into each portion of a segment. Each session included 12 basic (i.e., reinforced) trials. The position of probe trials in a session was random; however, each session began with four basic trials, and at least one basic trial separated each probe trial. For Group FT-Lever, the lever was presented in the same manner as during training. To remain consistent with Experiment 1, only data from the 1 st test day are shown.

The percentage of probe trials with a ball-bearing contact and the duration of nosing in the feeder during each quarter of the IFI were measured. The duration of contacting the lever was also measured. This response did not change significantly across the IFI for either group $[F \mathrm{~s}(3,21)<1]$, as in Experiment 1 . Therefore, the lever contact data are not shown.

\section{Results}

\section{Nosing in the Feeder Across \\ the FT Interval: Training Versus Test}

Figure 5 shows the duration per second spent nosing in the feeder averaged over the last four FT training sessions compared with the basic FT trials of the first test session for Groups FT-Time and FT-Lever. Subjects in both groups showed similar changes in nosing in the feeder: Nosing in the feeder increased over the last half of the IFI $[F \mathrm{~s}(3,21)>30.74]$. Scheffé tests conducted on data for each group confirmed that during the training and test sessions, nosing increased from $\mathrm{S} 2$ to $\mathrm{S} 4$ for both groups.

\section{Ball-Bearing Contact}

Figure 6 shows the percentage of trials with a ballbearing contact during each quarter of the IFI on the first test session for both groups. Ball-bearing contact did not differ among Segments 1, 2, 3, and 4 for either group $[F \mathrm{~s}(3,21)<1]$. Each group's pattern and level of ballbearing contact (data not shown) were similar for the remaining three test sessions.

\section{Ball-Bearing Versus Basic FT Trials}

Figure 7 compares nosing in the feeder on the 1st test day during the four segments in which ball bearings were presented (ball-bearing trials) and compares it with nosing in the feeder from the same interval during trials on which no ball bearing was presented (basic FT trials). For Group FT-Time, subjects spent less time nosing in the feeder during ball-bearing trials than during basic FT trials during the second and third segments $[t \mathrm{~s}(7)>2.25]$. For Group FT-Lever, subjects spent less time nosing in the feeder during ball-bearing trials than during basic FT trials during the second, third, and fourth segments $[t \mathrm{~s}(7)>2.65]$.

As shown in Figure 7, nosing in the feeder was suppressed during Segments 2 and 3 for Group FT-Time. Since nosing appeared slightly more suppressed during S2 than S3, suppression ratios were calculated. The ratios were obtained in the same manner as in Experiment 1. The suppression ratios for Segments 2 and 3 were .21 and 

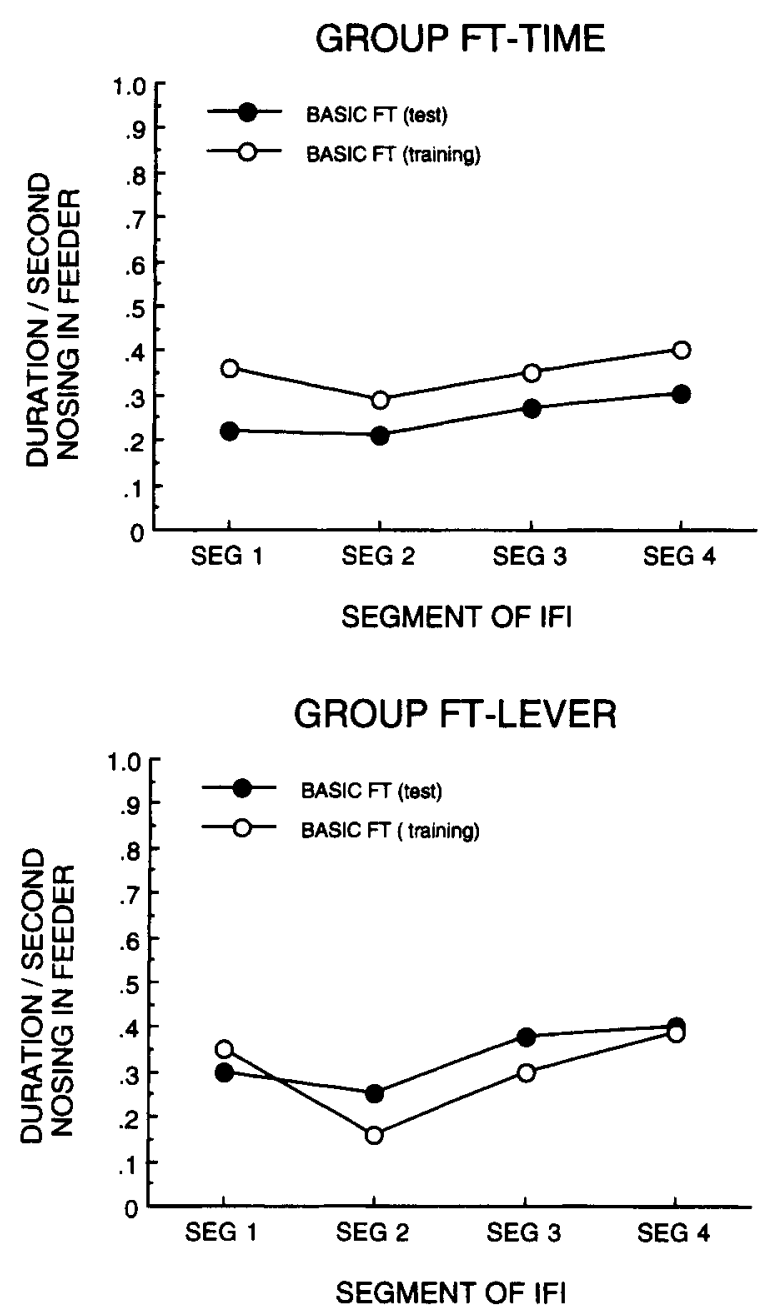

Figure 5. The mean duration per second spent nosing in the feeder averaged across the last 4 days of fixed-time (FT) training, and during the FT trials of the first test session for Groups FTTime and FT-Lever.

.24 , respectively, and the differences were not statistically significant $[t(7)<1]$. Suppression ratios for Segments 2 , 3 , and 4 of Group FT-Lever were .20, .19, and .22, respectively, and the differences were not statistically significant $[F(2,16)<1]$.

\section{Discussion}

Unlike in Experiment 1, unconditioned ball-bearing probes in the present experiment evoked relatively low contact when presented during each quarter of the IFI. This result suggests that the unsignaled passage of time alone was insufficient to strongly elicit the appropriate conditioned search mode and/or provide the cues necessary to differentially evoke the ball-bearing contact response. It may be that the evocation of the search state and ball-bearing response requires a better defined external stimulus, and also might profit from a pairing with food instead of the careful avoidance of pairing, as in the present probe procedure.
The rats in both groups of the present experiment developed a pattern of nosing in the feeder across the IFI that was similar to the pattern observed in Experiment 1. It might be argued that since rats displayed the same pattern of nosing whether the clock was present (Experiment 1 ) or absent (Experiment 2), the clock does not control this response. However, comparison of the absolute levels of nosing between Experiments 1 and 2 shows that the clock did have some control of nosing in the feeder: During the last half of the IFI, rats spent more time nosing in the feeder when the clock stimuli were present (Experiment 1) than when they were absent (Experiment 2). Although conclusions based on between-experiments comparisons must be made with caution, K. M. Silva and Timberlake (1998) explicitly examined the effect of the presence versus the absence of an interfood clock on responding and obtained the same effect (see also Palya \& Bevins, 1990, for similar results with pigeons).

In sum, the results from Experiments 1 and 2 together suggest that markedly elevated ball-bearing contact during the second quarter of the IFI is due to the presence of a well-defined external cue predicting food at a distance

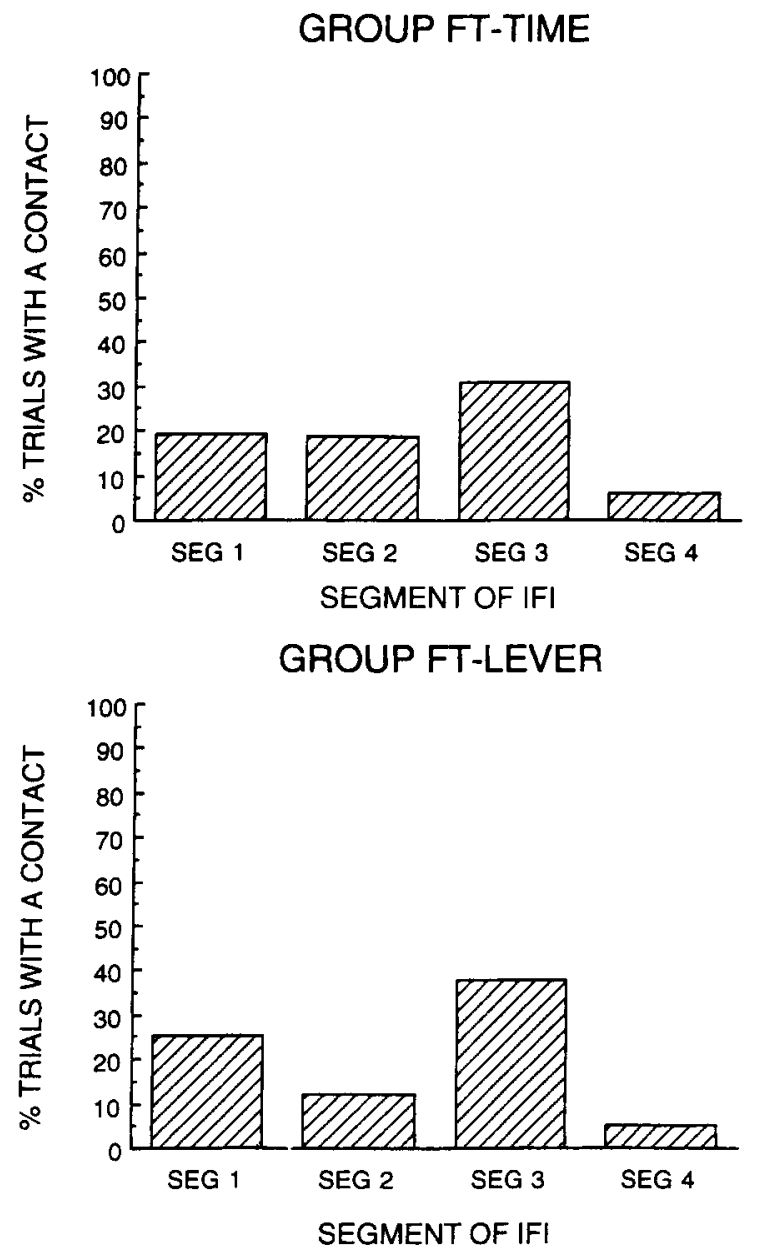

Figure 6. The percentage of trials with a ball-bearing contact during each quarter of the interfood interval (IFI) during test for Groups FT-Time and FT-Lever. 

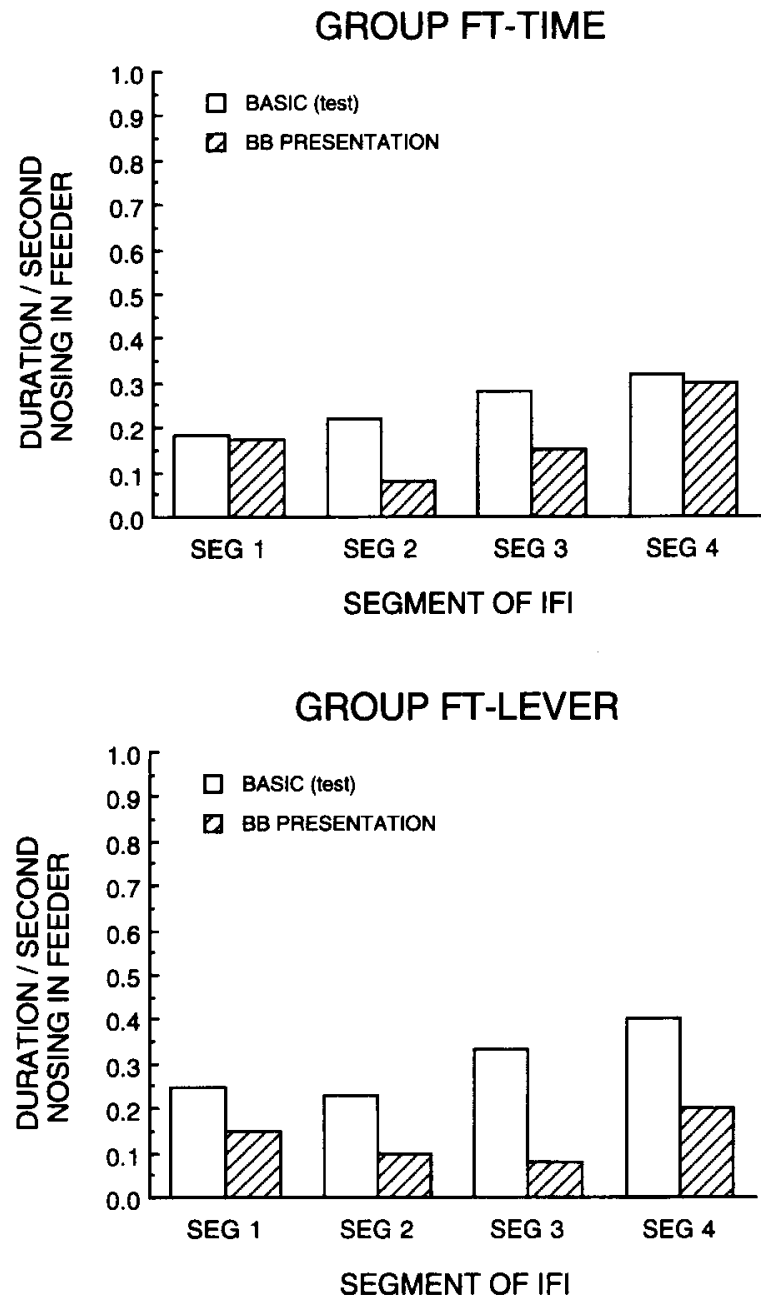

Figure 7. The mean duration per second spent nosing in the feeder from the portions of the interfood interval (IFI) in which ball bearings were presented (hatched bars) and from the same time period during basic fixed-time (FT) trials (solid bars) of the test. Because ball bearings were presented 4 sec after the beginning of each quarter of the IFI, the data shown were taken from the last 8 sec of each quarter.

(see also K. M. Silva \& Timberlake, 1998). In view of the results of Experiment 2, it would be interesting to know the outcome if less well-defined external cues were used. It has been argued elsewhere (Akins, Domjan, \& Gutierrez, 1994; Domjan, 1994; Timberlake \& Lucas, 1989) that responses occurring early in a behavioral sequence (more distant from food) may be more readily elicited by diffuse external cues than by more punctate cues. The latter presumably more strongly control responses later in the sequence. However, it may also be that cues with clear onsets and offsets are important in supporting the highest levels of unconditioned ball-bearing behavior. For example, discrete sounds, sights, and perhaps odors may set the stage for discovering and pursuing prey stimuli in natural settings (see, e.g., Tinbergen, 1951). Last, that ball-bearing contact was similar during all segments of the IFI for Group FT-Lever suggests that the lever had no significant effect on the pattern of ball-bearing contact observed in Experiment 1.

\section{GENERAL DISCUSSION}

Rats exposed to training with a four-segment interfood clock spent increasing amounts of time nosing in the feeder during the two clock stimuli prior to food. During a test in which a rolling ball bearing served as a probe for the strength of the general search mode, subjects contacted more than twice as many ball bearings when they were presented during a clock stimulus temporally far from food (S2) than during the other clock stimuli. A similar test in which a moving door was presented instead of the ball bearing showed no difference in approach and contact of the door among the different clock stimuli. A separate test of the importance of the external clock stimulus and the timing cues provided by periodic withdrawals and insertions of the lever revealed no effect of the latter, but indicated a critical importance for the external clock stimulus in facilitating contact of the ball-bearing probe. Control of contact with the ball bearing was apparently not attributable to the passage of time alone.

The data were not compatible with the argument that animals were particularly sensitive to the dimensions of novelty, movement, and sound during S2. If this had been the case, animals should have responded to the moving door as well as the ball bearing. Instead, it appeared that the stimulus configuration of the unconditioned ball-bearing probe was the critical determinant of the behavior directed toward it during $\mathrm{S} 2$.

The data also were not compatible with the argument that the pattern of contact with the ball bearing was caused by competition between contacting the ball bearing and nosing in the feeder. On this view, there should have been higher contact with the ball bearing during clock stimuli in which competition with nosing in the feeder was less, namely S1, S2, and S3. In general there should have been a negative relation between amount of nosing in the feeder and the degree of contact with the ball bearing. Further, the maximum level of contact with the ball bearing should have been approximately that shown in baselines of unconditioned ball-bearing contact (K. M. Silva \& Timberlake, 1997; Timberlake et al., 1982). Finally, the response competition argument predicts that contact with the ball bearing during $\mathrm{S} 2$ of the second experiment should have been similar to that occurring in Experiment 1. The patterns of nosing in the feeder were similar across the IFI in the two experiments, and therefore competition with ballbearing contact should have been approximately the same.

The data do not confirm any of the response-competition predictions. There was no systematic negative relation between ball-bearing contact and amount of nosing in the feeder during the clock stimuli in Experiment 1. Furthermore, Figure 4 shows that during basic trials of the test, nosing in the feeder was greatest during $\mathrm{S} 4$, lower during 
S3, even lower during S2 and S1. A competing behaviors account predicts that ball-bearing contact should be highest during $\mathrm{S} 1$ and $\mathrm{S} 2$, lower during S3, and lowest during S4. However, ball-bearing contact was greatest during S2 and was low during S1, S3, and S4.

Similarly, the level of ball-bearing contact during S2 was more than twice the baseline level, as predicted by the response-competition explanation. In fact, the level of ball-bearing contact during S2 was similar to levels observed when food presentations are made contingent on ball-bearing contact. Finally, in Experiment 2, subjects nosed in the feeder more during S4 than during S2 during training and test. From a competing behavior view, subjects should have had more time to interact with a ball bearing presented during S2 than S4. However, the results showed relatively low and similar levels of ball-bearing contact during all quarters of the IFI.

\section{Behavior Systems}

The results are consistent with a behavior systems view, which predicts that stimuli temporally far from food should come to control a general search mode and behavior related to locomoting, chasing, and contacting moving stimuli. In contrast, stimuli immediately before food should come to control focal search responses related to behavior directed at the imminent location of food (the food site). During initial training, a temporally distant clock stimulus (S2) conditioned very little nosing in the feeder (a more focal search response) relative to the two stimuli ( $\mathrm{S} 3$ and $\mathrm{S} 4$ ) presented before food presentations. However, during a test, subjects contacted more novel, unconditioned ball bearings when they were presented following the onset of S2 (a more general search response) than when they were presented following the onset of the other clock stimuli. That $\mathbf{S} 2$ facilitated contact with a rolling ball bearing and not contact with a moving door is consistent with the behavior systems assumption that the general search mode conditioned to temporally distant stimuli facilitates responses related to chasing moving objects of a particular size (Timberlake \& Washburne, 1989).

The evidence for the focal search mode controlled by the temporally proximate cues consisted of increased nosing in the feeder when the clock stimuli changed from S2 to S3 and from S3 to S4 (see also Dinsmoor, 1994; Palya \& Bevins, 1990; Palya \& Pevey, 1987). However, unlike the case for the general search mode, the assumption of a conditioned focal search mode is not necessary to account for these data. The nosing in the feeder data are also compatible with the simple conditioning of the response of nosing in the feeder as an inverse function of the temporal distance between the CS and US (Rescorla \& Wagner, 1972); these data are also compatible with the surmise that stimuli during the last half of the IFI provide positive information about the delivery of food (Gibbon \& Balsam, 1981). However, neither of these views predicts the form of the response.

However, despite the low levels of nosing in the feeder to $\mathrm{S} 2$ during initial interfood clock training, the measure of contact of the unconditioned ball bearing during the test suggests that some form of conditioning during the interfood clock training occurred during the more temporally distant clock stimulus (S2). In the test of Experiment 1, subjects contacted more ball bearings during S2 than they did during other clock stimuli presented closer to food.

The present results are also compatible with Palya's (1993) bipolar model, which specifies that within an IFI, a gradient extends from the stimulus most negatively correlated with the reinforcer to the stimulus most positively correlated with the reinforcer, and qualitative changes in behavior are expected to stimuli correlated with these various portions of the IFI. In this view, stimuli early in the IFI signal nonreinforcement, and as a result are aversive and function as negative reinforcers. However, stimuli late in the IFI signal reinforcement, and as a result function as positive reinforcers. Palya (1993) and Dinsmoor et al. (1986) provided evidence for this view by showing that stimuli correlated with successively later portions of the second half of the IFI support successively higher rates of response-contingent stimulus production, whereas stimuli in successively earlier portions of the first half of the IFI support higher rates of stimulus removal. The present results provide support for this model by showing that stimuli during the last half of the IFI controlled one response (nosing in the feeder), whereas stimuli near the beginning of the IFI controlled a qualitatively different response (ball-bearing contact). However, the present results also suggest that stimuli in the first half of the IFI may serve as positive reinforcers if the appropriate responses are measured. By relating behavior during an interfood clock to the rats' feeding system, the behavior systems view adds to the bipolar account by predicting the response form that occurs during different clock stimuli.

\section{Other Causal Factors}

Other causal contributors might be involved. For example, it could be argued that sensitization or habituation to the frightening aspects of the ball bearing produce high levels of ball-bearing contact during S2 (see K. M. Silva \& Timberlake, 1997). However, the results are inconsistent with this view. First, if this were the case, one might expect ball-bearing contact to increase with repeated ball-bearing presentations. However, there was no trend for rats to systematically increase ball-bearing contacts across the test trials in Experiment 1 or 2. Second, for these processes to differentially produce high levels of contact during S2, the S2 test trials would need to be presented at the end of the test session, when the effects of sensitization or habituation should have been greatest. However, this problem was avoided by ensuring that the probe trials were presented in random order. Furthermore, if sensitization and habituation were responsible for elevated ball-bearing contacts during S2, one might have expected these processes to produce systematic changes in cage contacts over test trials in Experiment 1. However, this did not occur either.

The behavior systems view posits that the general search mode conditioned to $S 2$ during training facilitated contact of the ball-bearing above levels during the other 
clock stimuli, but it might also be argued that the rats' high level of ball-bearing contact during $\mathrm{S} 2$ could have been a result of associative processes other than conditioning of a search mode to the distant CS. For example, because the test presented ball bearings in combination with the previously conditioned clock stimuli, it could be that the ball-bearing contact during $\mathrm{S} 2$ was a result of second-order conditioning of the ball bearing by the clock stimulus. However, the results are inconsistent with this explanation. According to this view, S4 should acquire the most associative strength by virtue of being contiguous with primary reinforcement (food). Associative strength should then diffuse back to the earlier stimuli so that each successively earlier stimulus has less associative strength than the one that follows it. If the amount of ball-bearing contact depends on the reinforcing strength of the clock stimulus with which it is paired, then ballbearing contact should have been highest during $\mathrm{S} 4$, lower during S3, even lower during S2, and lowest during S1. However, the amount of ball-bearing contact was highest during S2 and was similarly low during the other clock stimuli.

It also might be argued that context conditioning or inhibition of delay could account for the elevated levels of ball-bearing contact during S2. For example, comparator models of associative learning (Miller \& Schactman, 1985 ) would suggest that explicit stimuli at the beginning of the IFI gain little associative strength because they are relatively weak predictors of the US. As a result, the associative strength not accrued by these early stimuli is gained by the context. If it is assumed that ball-bearing contact was more controlled by the context than nosing in the feeder (see Domjan, 1994), this view predicts that rats should contact more ball bearings during the first half (S1 and S2) of the IFI than during the last half. However, Figures 3 and 6 show that elevated levels of contact occurred only during S2 of Experiment 1, in which explicit predictive stimuli were used. The results also do not support an inhibition of delay account (Pavlov, 1927), which argues that a temporally distant CS (e.g., S1 and S2) becomes inhibitory during training. A novel stimulus presented during $\mathrm{S} 1$ or $\mathrm{S} 2$ should then produce disinhibition, resulting in an increase in the CR. By this account, the rats should have spent more time nosing in the feeder during S1 and S2, when the ball bearing (a novel stimulus) was present, than when it was absent. However, the results showed that the novel stimuli decreased nosing in the feeder relative to when the ball bearing or door was absent (see also Palya \& Chu, 1996).

In conclusion, the present results are consistent with Timberlake and Lucas's (1989) behavior systems view that stimuli temporally far from food should come to control a general search mode and behavior related to chasing and contacting moving stimuli, whereas stimuli proximate to food should come to control focal search responses related to behavior directed at the food site (see also Domjan, 1994; Fanselow, 1994; Hogan, 1994; Timberlake, $1983,1994,1995)$. The present results also provide support for Palya's (1993) gradient of responding during an interfood clock. Consistent with this view, stimuli during the last half of the IFI controlled one response, whereas stimuli during the first half of the IFI controlled a qualitatively different response. However, by relating behavior during an interfood clock to the conditioning of a succession of search modes in the rat's feeding system, the behavior systems view adds to previous substitution, information, or associative interpretations (e.g., Matthews \& Lerer, 1987; Palya, 1993) by providing a framework that considers the feeding ecology of the subject and how it may fit with the search states, stimuli available, and resulting behavior.

\section{REFERENCES}

Akins, C. A., Domjan, M., \& Gutierrez, G. (1994). Topography of sexually conditioned behavior in male Japanese quail (Coturnix japonica) depends on the CS-US interval. Journal of Experimental Psychology: Animal Behavior Processes, 20, 199-209.

BARNETT, S. A. (1975). The rat: A study of behavior. Chicago: University of Chicago Press.

DinsmoOR, J. A. (1994). A comparison between the block clock and standard autoshaping procedures. Learning \& Motivation, 25, 313-337.

Dinsmoor, J. A., Dougan, J. D., Pfister, J., \& Thiels, E. (1992). The autoshaping procedure as a residual block clock. Journal of the Experimental Analysis of Behavior, 58, 265-276.

Dinsmoor, J. A., LeE, D. M., \& BRown, M. M. (1986). Escape from serial stimuli leading to food. Journal of the Experimental Analysis of Behavior, 46, 259-279.

Domjan, M. (1994). Formulation of a behavior system for sexual conditioning. Psychonomic Bulletin \& Review, 1, 421-428.

EWER, R. F. (1971). The biology and behavior of a free-living population of black rats (Rattus rattus). Animal Behavior Monographs, 4(3).

Fanselow, M. S. (1994). Neural organization of the defensive behavior system responsible for fear. Psychonomic Bulletin \& Review, 1, 429-438.

GibBon, J., \& BaLsam, P. D. (1981). The spread of association in time. In C. M. Locurto, H. S. Terrace, \& J. B. Gibbon (Eds.), Autoshaping and conditioning theory (pp. 219-253). New York: Academic Press.

HOGAN, J. A. (1994). Structure and development of behavior systems. Psychonomic Bulletin \& Review, 1, 439-450.

LuCas, G. A., Timberlake, W., \& GaWley, D. J. (1988). Adjunctive behavior of the rat under periodic food delivery in a 24 -hour environment. Animal Learning \& Behavior, 16, 19-30.

Matthews, T. J., Bordi, F., \& Depollo, D. (1990). Schedule-induced kinesic and taxic behavioral stereotypy in the pigeon. Journal of Experimental Psychology: Animal Behavior Processes, 16, 335-344.

MATTHEWS, T. J., \& LERER, B. E. (1987). Behavior patterns in pigeons during autoshaping with an incremental conditioned stimulus. Animal Learning \& Behavior, 15, 69-75.

Miller, R. R., \& Schactman, T. R. (1985). The several roles of context at the time of retrieval. In P. D. Balsam \& A. Tomie (Eds.), Context and learning (pp. 167-194). Hillsdale, NJ: Erlbaum.

PALYA, W. L. (1985). Sign-tracking with an interfood clock. Journal of the Experimental Analysis of Behavior, 43, 321-330.

Palya, W. L. (1993). Bipolar control in fixed interfood intervals. Journal of the Experimental Analysis of Behavior, 60, 354-359.

Palya, W. L., \& Bevins, R. A. (1990). Serial conditioning as a function of stimulus, response, and temporal dependencies. Journal of the $E x$ perimental Analysis of Behavior, 53, 65-85.

Palya, W. L., \& CHU, J. Y. M. (1996). Real-time dynamics in the interaction between a trial stimulus and its temporal context. Animal Learning \& Behavior, 24, 92-104.

Palya, W. L., \& Pevey, M. E. (1987). Serial conditioning as a function of parametric variations of an interfood clock. Animal Learning \& Behavior, 15, 249-262.

Papadouka, V., \& Matthews, J. T. (1995). Motivational mechanisms and schedule-induced behavioral stereotypy. Animal Learning \& Behavior, 23, 461-469. 
Pavlov, I. P. (1927). Conditioned reflexes (G. V. Anrep, Trans.). London: Oxford University Press.

RESCORLA, R. A., \& WAGNeR, A. R. (1972). A theory of Pavlovian conditioning: Variations in the effectiveness of reinforcement and nonreinforcement. In A. H. Black \& W. F. Prokasy (Eds.), Classical conditioning II: Current research and theory (pp. 64-99). New York: Appleton-Century-Crofts.

Silva, F. J., Timberlake, W., \& Koehler, T. L. (1996). A behavior systems approach to bidirectional excitatory serial conditioning. Learning \& Motivation, 27, 150-160.

Silva, K. M. (1996). Appetitive behavior during an interfood clock. Unpublished doctoral dissertation, Indiana University.

Silva, K. M., \& Timberlake, W. (1997). A behavior systems view of conditioned states during long and short CS-US intervals. Learning \& Motivation, 28, 465-490.

Silva, K. M., \& TimberLAKE, W. (1998). The organization and temporal properties of appetitive behavior in the rat. Animal Learning \& Behavior, 26, 182-195.

Staddon, J. E. R., \& Simmelhag, V. L. (1971). The "superstition" experiment: A re-examination of its implications for the principles of adaptive behavior. Psychological Review, 78, 3-43.

STEININGER, F. von (1950). Beiträge zur Soziologie und sonstigen Biologie der Wanderatte. Zeitschrift für Angewandte Zoologie, 7, 356-379.

TELLE, J. J. (1966). Beiträge zur Kenntnis der Verhaltensweise von Ratten, vergleichend dargestellt bei, Rattus norvegicus und Rattus rattus. Zeitschrift für Angewandte Zoologie, 48, 97-129.

TIMBERLAKE, W. (1983). The functional organization of appetitive behavior: Behavior systems and learning. In M. D. Zeiler \& P. Harzem (Eds.), Advances in analysis of behavior: Vol. 3. Biological factors in learning (pp. 177-221). Chichester, U.K.: Wiley.

TimberlaKe, W. (1994). Behavior systems, associationism, and Pavlovian conditioning. Psychonomic Bulletin \& Review, 1, 405-420.
Timberlake, W. (1995). Reconceptualizing reinforcement: A causal-system approach to reinforcement and behavior change. In W. O'Donohue \& L. Krasner (Eds.), Theories of behavior therapy: Exploring behavior change (pp. 50-96). Washington, DC: American Psychological Association.

Timberlake, W., \& Grant, D. L. (1977). Autoshaping in rats to the presentation of another rat predicting food. Science, 190, 690-692.

Timberlake, W., \& LuCAS, G. (1985). The basis of superstitious behavior: Chance contingency, stimulus substitution, or appetitive behavior. Journal of the Experimental Analysis of Behavior, 44, 279-299.

TIMBERLAKE, W., \& LuCAS, G. (1989). Behavior systems and learning: From misbehavior to general principles. In S. B. Klein \& R. R. Mower (Eds.), Contemporary learning theories: Instrumental conditioning theory and the impact of biological consiraints on learning (pp. 237275), Hillsdale, NJ: Erlbaum.

Timberlake, W., \& Silva, K. M. (1995). Appetitive behavior in psychology, ethology, and behavior systems. In N. Thompson (Ed.), Perspectives in ethology: Vol. 11. Behavioral design (pp. 212-254). New York: Plenum.

Timberlake, W., Wahl, G., \& King, D. (1982). Stimulus and response contingencies in the misbehavior of rats. Journal of Experimental Psychology: Animal Behavior Processes, 8, 62-85.

Timberlake, W., \& Washburne, D. L. (1989). Feeding ecology and laboratory predatory behavior toward live and artificial moving prey in seven rodent species. Animal Learning \& Behavior, 17, 2-11.

TINBERGEN, N. (1951). The study of instinct. Oxford: Ox ford University Press, Clarendon Press.

(Manuscript received July 21, 1997; revision accepted for publication March 24, 1998.) 